\title{
Contrast Enhancement of Images through Skewness and Mode Based Bi-Histogram Equalization
}

\author{
Kuldip Acharya \\ Department of Computer Science and Engineering, National Institute of Technology, Agartala, Barjala, Jirania, Tripura \\ (West), Pin: 799046, India \\ Email: kuldip.acharjee@gmail.com
}

\section{Dibyendu Ghoshal}

Department of Electronics and Communication Engineering, National Institute of Technology, Agartala, Barjala, Jirania, Tripura (West), Pin: 799046, India

Email: tukumw@gmail.com

Received: 25 February 2020; Accepted: 02 July 2020; Published: 08 October 2020

\begin{abstract}
In this paper, skewness and mode-based histogram equalization algorithm have been proposed for contrast enhancement of digital images. The present method gives a novel idea for histogram clipping and histogram bifurcation. The prior is done with the skewness value and the latter is done with help of mode values of the intensity level random data set. The pixel intensity levels are random and thus a stochastic approach has been used and found to yield improved figure of merits. The image histogram has been clipped with the help of a pre-assigned threshold value computed from skewness value to restrict the rate of over enhancement. The clipped histogram is subdivided into two parts, using the histogram subdivision limit which is calculated on the basis of the mode value of the image. Histogram of individual sub-image is equalized independently and then integrated to form the final enhanced image. The simulation results have shown that the proposed skewness and mode based bi-histogram equalization algorithm enhances the contrast of the image in a better manner compared with the other histogram equalization methods in terms of FSIM, PSIM, SFF, VSI, HaarPSI, and GMSD.
\end{abstract}

Index Terms: Skewness, Mode, Contrast, Enhancement, Bi-histogram.

\section{Introduction}

Image enhancement is done for better visualization, image understanding and subsequent analysis [1]. An image histogram is obtained to find the number of pixels occupying either a particular or certain range of intensity levels in a digital image. The histogram is then processed in order to alter the pixels numbers to occupy equally or almost equally space gray events and this leads to enhance the contrast of the image. It is often found that histogram processing of all the pixels is difficult and the task becomes easier when the total intensity levels are divided into certain portions. There should be a basis for divisions of both color and gray levels. The standard image processing methods cover image enhancement, image restoration, image compression, segmentation, morphological processing, pattern recognition and histogram equalization [1]. Chen and Ramli proposed minimum mean brightness error bi-histogram equalization (MMBEBHE) for preserving the mean brightness "optimally" [2]. MMBEBHE is an improvement of BBHE algorithm, which computes the absolute mean brightness error (AMBE) for gray levels 0 to L-1 and intersects the histogram based on the intensity value, which gives minimum AMBE. Effective image enhancement methods for fog-affected indoor and outdoor images were proposed by Kim et al. [3] founded on contrast limited adaptive histogram equalization and combination of dark channel prior (DCP) through discrete wavelet transform (CLAHE-DWT) procedures. He Deng et al. [4] proposed a new image enhancement method on intuitionistic fuzzy sets, and defuzzification processes on each zone to improve the visual quality of the region of interest (ROI). Images acquired under poor light circumstances have low contrast and darkness is observed on image regions. Yongbin Zhang et al. [5] proposed an enhancement method of images based on dynamical stochastic resonance (DSR) for non-uniform illumination image enhancement. Monostable Langevin equation is used to enhance the low-contrast images. Plateau limit-based tri-histogram equalization algorithm for image enhancement was proposed by A. Paul et al. [6]. The plateau limit is computed from the mean and the median intensity values for better image enhancement. Night time image enhancement using a new illumination boost algorithm was proposed by Zohair Al-Ameen et al. [7] for improving the low brightness, poor contrast and faded color of low 
light images appropriately. An image enhancement technique is proposed by Chaoying Tang et al. [8] where initially, the bright channel prior is used to the inverted image to fade the strong light and then, a dehazing process through the dark channel prior is applied through super pixel segmentation method to improve the low-light image. Lastly, a nonlocal denoising technique is followed for further enhancement. Triple clipped dynamic histogram equalization based on the standard deviation method for contrast enhancement is proposed by Majid Zarie et al. [9]. This method shows a natural enhancement by creating vibrant images through maximum details. Contrast-based image enhancement algorithm using grey-scale and color space was proposed by Amita Nandal et al. [10]. RGB images were enhanced by fine-tuning of brightness and contrast to achieve a visually pleasing image. The method used hue saturation value (HSV) and contrast enhancement (CE) to attain enhanced-color images. The images combined in RGB color space may be affected by the dark contrast but in HSV color space, greyscale information is maintained in separate channels and the combined images were not affected by the similar dimming contrast. This technique involved a calculation of mapping function, gradient-based function, and eigenvalue computation was utilized for contrast enhancement. Tuned trithreshold fuzzy intensification operators (TTFIO) [11] approach used a fuzzy membership function that set the pixels values of a given channel to a range between 0 and 1 which gave better color quality. The multiscale Retinex method was extensively utilized for image enhancement on constraints such as Gaussian scales, gain and offset, etc. An advanced Retinex algorithm based on PSO was used to tune the constraints for multiscale Retinex with chromaticity preservation (MSRCP) [12] which achieved an improved result from other approaches. Jobson et al. proposed singlescale Retinex (SSR), Multiscale Retinex (MSR) and Multiscale Retinex with color (MSRCR) found on Land's Retinex [13]. In SSR, a center-bounded algorithm, the lighting was measured through the variance among the input value of the center and an average of its neighborhood. MSR method could avoid the unnatural appearance seen in the SSR method and can produce satisfactory exchange among dynamic range compression and color rendering. Three fidelity principles and numerical approaches were proposed by MSRCR by Yuhong Liu et al. [14] to estimate the reliability of image enhancement. The outcome showed that the MSRCR technique could produce more falsehood, particularly for overdark and over-bright images. An adaptive geometric filter (AGF) method based on the average intensity of the image and discrete cosine transform (DCT) coefficient adjustment [15] is proposed by Kansal et al. for gray and color image enhancement. AGF is employed for smoothing the histogram peaks before histogram equalization and DCT coefficient adjustment is done to enhance the finer details of the image. The proposed method is a statistical method dealing with the adjustments of pixel numbers having a particular value or certain range of random intensity levels. Histogram equalization method is a well-known and established technique in the field of image enhancement. Although a number of techniques for histogram equalization has been reported by various scientists, but no study for histogram equalization dealing with clipping of histogram using skewness value and bifurcation of pixel intensity levels has not been found by the present author despite surveying best resources available with them. Besides, all the related parameters have been found to yield improved values compared with the earlier studies. The output images have been found to possess better visual quality as well as improved quantitative parameters. The study carries further weight as the proposed algorithm has been found to be effective for low contrast images. The major research objective was to explore new possible ideas associated with random parameters. The pixel intensity levels are random and the number of pixels need to be manipulated in order to have histogram equalization. Some rationale was required to be found out. The present authors attempted to explore all such stochastic approach-based possibilities which could yield better output from subjective and objective evaluation points of view. The aim of the study was to found a novel approach dealing with statistical method to tackle random data related problem. The problem was how to enhance various types of image, especially low contrast images in better ways. The superiority of the proposed method would be established through the output parameters related to image enhancement. According to the knowledge resources available with the present author, the present algorithm can be considered to be the best for enhancing low contrast images based on the concept of histogram equalization.

The authors intend to propose novel concept for enhancement of low contrast images and it should be related to histogram equalization. Histogram equalization is a statistical approach dealing with random intensity levels of the pixels. Reported literature indicated myriad number of derived studies from basic histogram equalization and specification and these has got vast potential for further improved studies. The aim of the study has been seemingly fulfilled and which are spelt out from the output tables and images.

\section{A. Contributions of the suggested method}

In this paper, skewness and mode based bi-histogram equalization algorithms for image enhancement has been proposed. The proposed method utilizes the skewness of the color levels of RGB images and gray levels images to set the clipping limit of the histogram to restrict the over enhancement of the image. The mode value is used to separate the input histogram into two parts. Sub histograms thus obtained are sequentially processed and combined to achieve a single continuous histogram.

This paper is organized as follows: Section 2 presents the literature review and algorithm. Section 3 describes the proposed skewness mode based bi-histogram equalization method. Section 4 gives simulation results and explanation, and finally, the conclusion is given in Section 5 of the paper. 


\section{Literature Review}

In this section, a brief literature review works related to the proposed method has been described. Recursive HE algorithm (RESIHE) was introduced by Singh et al. for the enhancement of low exposure images [16]. It is observed in RESIHE algorithm that both the lower mean intensity pixels and higher mean intensity pixels are unable to produce adequate contrast enhancement. Besides, the method based on the recursive division often fails to give realistic output and may create extra artifacts in the border areas of image partition. Median mean-based sub-image clipped HE (MMSICHE) was suggested by Singh et al. [17]. In this method, a histogram of the image was subdivided into four sub histograms with the help of the mean and median of the image. Histogram was clipped by the median intensity of image and sub-images were equalized separately. Images gained from MMSICHE method may comprise artifacts and over enhancements. Skewness, an odd order statistic, is very useful to collect various information about reflectance and brightness of an image and the human visual system (HVS) is sensitive to it. Calculation of skewness is found easier with the help of HVS. Dark glossy surfaces possess high skewness and whitish matte surfaces have low or negative skewness. It is also found that the computation of local skewness with filters and neural mechanisms is easy [18]. The skewness of the luminance histogram is correlated with the reflectivity and gloss of any real-life surfaces. Visual assessment of lightness and glossiness is also correlated with luminance skewness. [19].

Most of the algorithm utilizes the mean or median value of the data set comprising the intensity levels in the histogram as a clipping limit and standard deviation to divide the histogram. In the proposed algorithm the histogram is clipped with a threshold limit calculated by the skewness value and mode values have been used to separate the input histogram. The statistical method has been applied in the present algorithm and the pixel intensity value which is larger than the clipping threshold value is deleted. Here, most of the pixels are set below the clipping limit and this has made interval value high. After that, each histogram of sub-images is equalized independently and all sub-images are integrated together to form an output image. The proposed research method is simple and corresponding Matlab coding is simple and easy to implement. The program run time is found to be small and thus can be considered efficient. The research methods have helped immensely to achieve image enhancement by using a novel idea associated with histogram equalization. The efficiency and efficacy have been established through improved output metrics compared with earlier research works. The research objective is thereby fulfilled.

\section{Proposed algorithm}

- The histogram is clipped in the present study with the help of a certain threshold limit that comes from the value of the skewness of occupied discrete intensity levels. The proposed algorithm provides simplicity to compute the threshold value through skewness intensity value and it is used to select the clipping limit of a histogram of the image under test.

- The histogram is divided into two parts by using sub histogram limit parameter $\mathrm{X}_{\mathrm{p}}$, which is based on the mode value of the data set constituting the image. In the proposed algorithm, a middle portion of the sub histogram covers a significantly large number of pixels and it enables to maintain the brightness of the image. Here, histogram parts of the image are equalized in an automated manner and finally merged to produce the output image.

The performance of the proposed algorithm is simulated and demonstrated over a number of images of the publicly available BSD dataset [20]. Comparative study of the present results have also been reported with respect to the other conventional algorithms using image quality metrics such as feature similarity index metric (FSIM) [21], perceptual similarity measure (PSIM) [22], sparse feature fidelity (SFF) [23], visual saliency-induced index (VSI) [24], The Haarwavelet based perceptually similarity index (HaarPSI) [25], and gradient magnitude similarity deviation (GMSD) [26].

\section{Proposed Methodology}

Histogram equalization (HE) is a traditional method of contrast enhancement of digital images. The proposed method is described in the following three sub-sections. Here, the bi-histogram of the image is processed by statistical operation for contrast enhancement. At first, histogram clipping is carried out with the help of skewness value, and then the sub histogram separation parameter is calculated by mode value. Finally, histogram subdivision and equalization are implemented to produce the output contrast enhancement image.

\section{A. Skewness based histogram clipping}

Histogram clipping is a process to restrict over enhancement effect to have a normal appearance of the image. Here, histogram bins are restricted to the threshold value calculated from skewness when their values are larger than the skewness threshold limit. Clipping threshold is a small number derived stochastic treatment of random data, i.e., the intensity level of the pixels.

Histogram of the image is signified as $\mathrm{H}(\mathrm{i})$ shown in 1 where $\mathrm{N}_{\mathrm{L}}$ is the total number of pixels and $\mathrm{L}$ represents total image intensity level. 


$$
\mathrm{N}_{\mathrm{L}}=\sum_{i=0}^{L-1} H(\mathrm{i})
$$

The mean intensity level calculates average number of image intensity levels which is denoted by $\overline{X_{\varphi}}$ and a standard deviation of image is denoted by $\sigma$ in 2 and 3, respectively.

$$
\begin{gathered}
\overline{X_{i}}=\frac{\sum_{i=0}^{L-1} i . H(\mathrm{i})}{\sum_{i=0}^{L-1} H(\mathrm{i})} \\
\sigma=\sqrt{\frac{\sum_{i=0}^{L-1}\left(\mathrm{i}-\overline{X_{i}}\right)^{2} \times H(\mathrm{i})}{\sum_{i=0}^{L-1} H(\mathrm{i})}}
\end{gathered}
$$

A clipped histogram is calculated by Skewness $\left(\mathrm{T}_{\mathrm{h}}\right)$ using the value of mean and standard deviation in 4 as

$$
\mathrm{T}_{\mathrm{h}}=\frac{\sum_{i=0}^{L-1}\left(X_{i}-\overline{X_{i}}\right)^{3} N \times H(\mathrm{i})}{\left(N_{L}\right) \sigma^{3} \times H(\mathrm{i})}
$$

$X_{i}$ is an image intensity value. $\mathrm{N}$ is pixel count and $N_{L}$ is the maximum image intensity level. The total number of pixels.

Where $H(i)$ original histogram of image i.

$T_{h}$ denotes threshold value calculated from skewness.

$$
C_{h}(i)=\left\{\begin{array}{l}
T_{h} \quad \text { if } H(i) \geq \mathrm{T}_{h} \\
H(i) \text { if } H(i)<T_{h}
\end{array}\right.
$$

$C_{h}(i)$ denotes clipped histogram.

Skewness acts as a third-order moment value in the treatment of stochastic data and skewness indicates the overall appearance of the image either towards the blackness or whiteness. Hence calculation of the clipping threshold can be treated as good as those derived on the basis of mean or median. Besides, skewness provides better insight regarding data location and this will, in turn, help in image enhancement.

The histogram clipping threshold value computation by skewness and histogram separation process by mode value is shown in Fig. 1

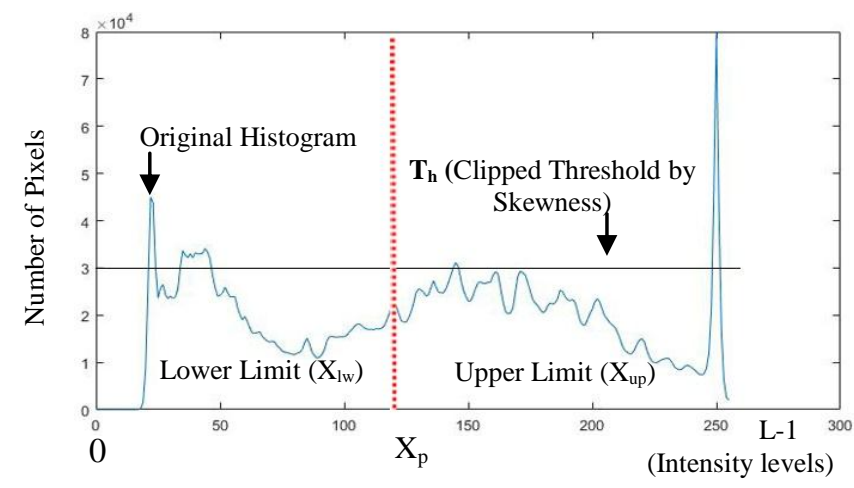

Fig. 1. Histogram Sub Division and Skewness based clipping process. 


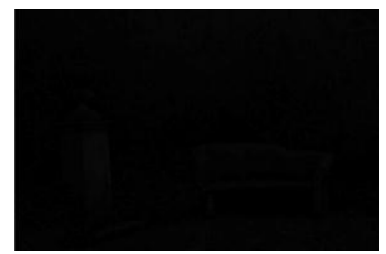

(a)

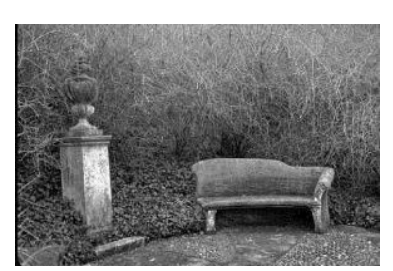

(c)

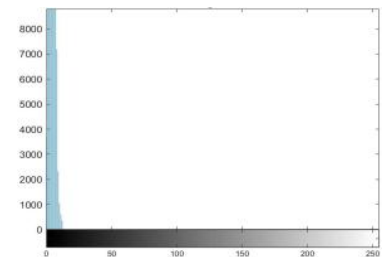

(b)

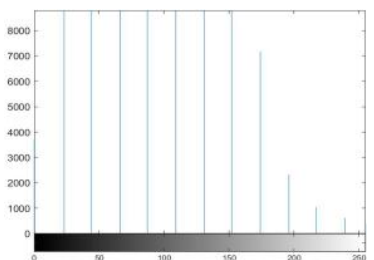

(d)

Fig.2 (a) Original Image, (b) Histogram of Original Image, (c) Processed Image, (d) Histogram of Processed Image.

\section{B. Mode based histogram sub division limit parameters}

Histogram can be separated with respect to any meaningful statistical parameter. In the earlier studies [16, 17] histogram division is done through the values obtained from the standard deviation. In the proposed method the original histogram is first bisected based on mode value $X_{p}$ as calculated in (6). The histogram subdivision method gives two sub-images $X_{l w}$ and $X_{u p}$ which range from intensity level 0 to $X_{p}$ and $X_{p}+1$ to L-1. L is a total number of intensity levels in the image. $X_{\mathrm{lw}}$ and $\mathrm{X}_{\mathrm{up}}$ be defined as a lower boundary and upper boundary sub-images, respectively. $\mathrm{X}_{\mathrm{p}}$ is a histogram separation value calculated by using the mode as stated in Eq. (6).

$$
X_{p}=\operatorname{Max}[\mathrm{H}(\mathrm{i})]
$$

Here, Max implies maximum, and it returns the value that occurs mostly from image histogram H(i).

The lower limit and upper limit of image histogram is calculated in (7) and (8).

$$
\begin{gathered}
X_{l w}=X_{0}+X_{p} \\
X_{u p}=X_{L-1}-X_{p}
\end{gathered}
$$

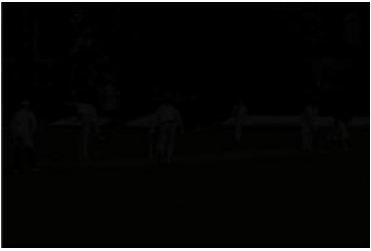

(a)

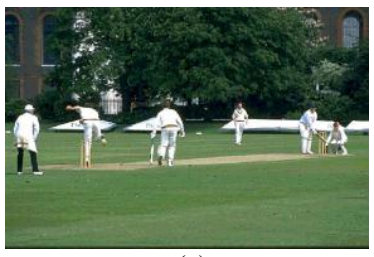

(c)

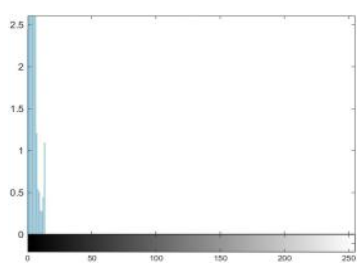

(b)

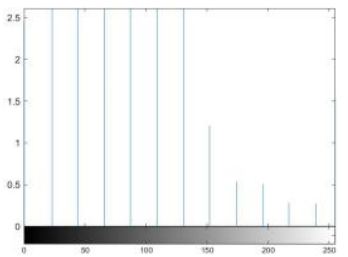

(d)

Fig.3 (a) Original Image, (b) Histogram of Original Image, (c) Processed Image, (d) Histogram of Processed Image.

$X_{l w}$ is a lower limit of histogram and $X_{u p}$ is an upper limit of the histogram. The mode is a stochastic parameter related to random data set and its value generally lies around the mean and median value. Thus, the separation of histogram on the basis of mode value is justifiable even it offers slight asymmetry in the separation of the data sets. Fig. 2(a), 2(b) shows an original grayscale image and its histogram and 2(c) and 2(d) show the enhanced image and its histogram by 
the proposed method. Fig.3(a), 3(b) shows the original color image and its histogram and 3(c) and 3(d) show the enhanced version of the image and its histogram by the proposed methodology. The proposed method of histogram clipping takes lesser time and found to be computationally efficient.

Table 1 FSIM outcomes of different algorithms

\begin{tabular}{|c|c|c|c|c|c|c|c|}
\hline BSD IMAGE & RESIHE & MMSICHE & SSR & MSRCR & MSRCP & Fuzzy & Proposed \\
\hline 10081 & 0.96353 & 0.9658 & 0.90712 & 0.89463 & 0.69705 & 0.53816 & 0.99159 \\
\hline 202000 & 0.96661 & 0.92508 & 0.8666 & 0.86435 & 0.76377 & 0.44466 & 0.99492 \\
\hline 202012 & 0.98965 & 0.97882 & 0.79434 & 0.78936 & 0.77158 & 0.18668 & 0.99511 \\
\hline 216066 & 0.93458 & 0.94742 & 0.87616 & 0.8843 & 0.78013 & 0.39116 & 0.9958 \\
\hline 226022 & 0.85967 & 0.95059 & 0.87104 & 0.81167 & 0.717 & 0.42981 & 0.99312 \\
\hline 299086 & 0.93856 & 0.9013 & 0.97275 & 0.94558 & 0.81669 & 0.57604 & 0.99197 \\
\hline 361010 & 0.87984 & 0.86258 & 0.85424 & 0.84578 & 0.76327 & 0.48412 & 0.99392 \\
\hline 37073 & 0.74285 & 0.81457 & 0.93897 & 0.93815 & 0.79261 & 0.5805 & 0.99609 \\
\hline Average & 0.90941125 & 0.91827 & 0.8851525 & 0.8717275 & 0.7627625 & 0.45389125 & 0.994065 \\
\hline
\end{tabular}

C. Histogram subdivision and equalization

a. Probability density function $(P D F)$

$\mathrm{P}_{\mathrm{lw}}$ (i) and $\mathrm{P}_{\text {up }}$ (i) are corresponding PDF of the sub images as follows:

$$
\begin{gathered}
P_{l w}(i)=\frac{C_{h}(i)}{N_{L w}} \text { for } 0 \leq \mathrm{i} \leq \mathrm{X}_{\mathrm{p}} \\
P_{l u}(i)=\frac{C_{h}(i)}{N_{L u}} \text { for } \mathrm{X}_{\mathrm{p}+\mathrm{i}} \leq \mathrm{i} \leq \mathrm{L}-1
\end{gathered}
$$

Where $\mathrm{N}_{\mathrm{Lw}}$ and $\mathrm{N}_{\mathrm{Lu}}$ are a total number of pixels in sub-images in the lower $\left(\mathrm{X}_{\mathrm{lw}}\right)$ and upper histogram $\left(\mathrm{X}_{\mathrm{up}}\right)$, respectively.

\section{Cumulative Density Function (CDF)}

$\mathrm{C}_{\mathrm{lw}}$ and $\mathrm{C}_{\mathrm{up}}$ are the corresponding $\mathrm{CDF}$ of individual sub-images and CDFs can be defined as

$$
\begin{aligned}
& C_{l w}(i)=\sum_{i=0}^{X_{p}} P_{l w}(\mathrm{i}) \\
& C_{u p}(i)=\sum_{i=X_{p+1}}^{L-1} P_{u p}(\mathrm{i})
\end{aligned}
$$

The next step of is to equalize both the sub histograms individually. The transfer function for histogram equalization based on equation (13) and (14) can be defined as

$$
\begin{gathered}
\mathrm{T}_{\mathrm{l}}=\mathrm{X}_{\mathrm{p}} \times \mathrm{C}_{\mathrm{lw}} \\
\mathrm{T}_{\mathrm{u}}=\left(\mathrm{X}_{\mathrm{p}}+1\right)+\left(\mathrm{L}-\mathrm{X}_{\mathrm{p}}+1\right) \mathrm{C}_{\mathrm{up}}
\end{gathered}
$$

$\mathrm{T}_{1}$ and $\mathrm{T}_{\mathrm{u}}$ are transfer functions utilized for equalizing the sub histograms individually. The next step is to merge both sub-images into one final image. The final output image is produced by a combination of both transfer functions.

\section{Simulation Results and Analysis}

Performance parameters are computed and determined over a number of images that are publicly available in the BSD (Berkeley Segmentation Database) image dataset [19]. All the algorithms are executed on a Windows 10 Pro with Intel Core i7 processor at $2.30 \mathrm{GHz}$ and $8 \mathrm{~GB}$ random access memory. The algorithms are implemented in MATLAB R2018a [27] with the support of default constraints specified in the specific algorithms. Visual quality observation and performance evaluation are then carried out on the output images. From a thorough comparative study, it is found that the proposed method is better than many other prevailing algorithms such as feature similarity index metric (FSIM) [21] perceptual similarity measure (PSIM) [22], sparse feature fidelity (SFF) [23], visual saliency-induced index (VSI) [24], 
The Haar-wavelet based perceptually similarity index (HaarPSI) [25] and gradient magnitude similarity deviation (GMSD) [26]. Another point to take note that the present algorithm is computationally simple and it demonstrates an image enhancement method based on a simple stochastic approach that keeps both the contrast and color quality of images at an optimal level.

\section{Quality Assessment of Enhanced Image}

To assess the visual quality of images by different methods, the following image quality metrics are evaluated.

\section{A. Feature similarity index metric (FSIM)}

Feature similarity index metric (FSIM) measures image quality and proposed by Zhang [21]. FSIM is shown in equation 15. FSIM computes the feature similarity between the input image and the output image. Here, original and enhanced image are represented by $f_{l}$ and $f_{2}$, respectively. Phase congruency (PC) for $f_{1}(\mathrm{y})$ and $f_{2}(\mathrm{y})$ are represented as $\mathrm{PC}_{1}$ and $\mathrm{PC}_{2}$, respectively. $S_{L}(\mathrm{y})$ specifies local feature similarity of $f_{1}(\mathrm{y})$ and $f_{2}(\mathrm{y})$ at pixel position $y$. $\mathrm{PC}_{m}(y)$ is computed as the maximum of $\mathrm{PC}_{1}(y)$ and $\mathrm{PC}_{2}(y)$.

Table 2 PSIM outcomes of different algorithms

\begin{tabular}{cccccccc}
\hline BSD IMAGE & RESIHE & MMSICHE & SSR & MSRCR & MSRCP & Fuzzy & Proposed \\
\hline 10081 & 0.99937 & 0.99944 & 0.99779 & 0.99694 & 0.99358 & 0.98667 & 0.99993 \\
202000 & 0.99947 & 0.99847 & 0.99741 & 0.99773 & 0.99524 & 0.98793 \\
202012 & 0.99982 & 0.9995 & 0.99636 & 0.99741 & 0.99391 & 0.99266 \\
216066 & 0.99917 & 0.99908 & 0.99735 & 0.99799 & 0.99536 & 0.99131 \\
226022 & 0.99815 & 0.99927 & 0.99747 & 0.99655 & 0.99372 & 0.98665 & 0.9999994 \\
299086 & 0.99891 & 0.99822 & 0.99664 & 0.99752 & 0.99692 & 0.98984 \\
361010 & 0.99772 & 0.99718 & 0.99744 & 0.99637 & 0.99572 & 0.98777 & 0.99997 \\
37073 & 0.99691 & 0.99723 & 0.99884 & 0.99884 & 0.99773 & 0.98922 & 0.999996 \\
Average & $\mathbf{0 . 9 9 8 6 9}$ & $\mathbf{0 . 9 9 8 5 4 8 7 5}$ & $\mathbf{0 . 9 9 7 4 1 2 5}$ & $\mathbf{0 . 9 9 7 4 1 8 7 5}$ & $\mathbf{0 . 9 9 5 2 7 2 5}$ & $\mathbf{0 . 9 8 9 0 0 6 2 5}$ & $\mathbf{0 . 9 9 9 9 2 7 5}$ \\
\hline
\end{tabular}

FSIM metric among images $f_{1}$ and $f_{2}$ is determined as

$$
\mathrm{FSIM}=\frac{\sum_{y \in \Omega} S_{L}(\mathrm{y}) \cdot \mathrm{PC}_{m}(y)}{\sum_{y \in \Omega} \mathrm{PC}_{m}(y)}
$$

The entire image is allocated via $\Omega$ to the spatial domain. From the analysis of simulation results obtained from FSIM shown in Table 1, it is observed that the proposed method gives the highest FSIM values for all the images. The average FSIM value of the proposed method is 0.994065 , shown in the bold italic face which demonstrates the best outcome with respect to the other algorithms. The second-best result for average FSIM is given by the MMSICHE algorithm which gives average FSIM value as 0.91827 , is designated by underline.

Table 3 SFF outcomes of different algorithms

\begin{tabular}{cccccccc}
\hline BSD IMAGE & RESIHE & MMSICHE & SSR & MSRCR & MSRCP & Fuzzy & Proposed \\
\hline 10081 & 0.9955 & 0.99503 & 0.94316 & 0.94729 & 0.36034 & 0.029938 & 0.99888 \\
202000 & 0.98708 & 0.98558 & 0.93021 & 0.95486 & 0.9065 & 0.63467 & 0.99854 \\
202012 & 0.99711 & 0.99601 & 0.95557 & 0.89845 & 0.82485 & 0.72453 \\
216066 & 0.94519 & 0.92078 & 0.97212 & 0.91748 & 0.90571 & 0.63155 \\
226022 & 0.92223 & 0.98243 & 0.80713 & 0.93477 & 0.33158 & 0.17298 & 0.99867 \\
299086 & 0.97098 & 0.9281 & 0.7722 & 0.75008 & 0.87706 & 0.67231 \\
361010 & 0.90564 & 0.94909 & 0.91017 & 0.92305 & 0.81896 & 0.6806 & 0.99728 \\
37073 & 0.88275 & 0.95037 & 0.85261 & 0.84656 & 0.79793 & 0.67357 & 0.99703 \\
Average & $\mathbf{0 . 9 5 0 8 1}$ & $\mathbf{0 . 9 6 3 4 2 3 7 5}$ & $\mathbf{0 . 8 9 2 8 9 6 2 5}$ & $\mathbf{0 . 8 9 6 5 6 7 5}$ & $\mathbf{0 . 7 2 7 8 6 6 2 5}$ & $\mathbf{0 . 5 2 7 5 1 8 5}$ & $\mathbf{0 . 9 9 7 7 9}$ \\
\hline
\end{tabular}

\section{B. PSIM: Perceptual similarity (PSIM) measure}

PSIM is a novel and fast perceptual based IQA metric proposed by K. Gu et.al [22] and shown in 16. PSIM metric combines the gradient magnitude similarities at two scales, the color information similarity, and a reliable perceptualbased pooling. 


$$
\operatorname{PSIM}=\frac{H^{\nu}}{L^{\omega}} * S^{\kappa} * C^{o}
$$

Here $v, \omega, \kappa, o$ are fixed parameters.

$\mathrm{L}^{\omega}$ calculated for the image gradient magnitude $(\mathrm{GM})$ similarity at a large scale. $\mathrm{S}^{\mathrm{K}}$ computed for image gradient magnitude (GM) similarity at a small scale. $\mathrm{C}^{\mathrm{o}}$ calculated for color information similarity of image. $\mathrm{H}^{\mathrm{v}}$ considered for high distortion-based pooling, more detail in [22].

It is observed from the investigation of PSIM values gained in Table 2, that the proposed method gives the highest PSIM values for all images. The average PSIM value shown in bold italic face of the proposed method is 0.9999275 , which demonstrates the best outcome with respect to the other algorithms. The second-best result for average is given by RESHIE algorithm which gives average PSIM value as 0.99869 , is indicated by underline.

\section{Sparse Feature Fidelity for Perceptual Image Quality Assessment (SFF)}

SFF is a novel FR IQM named sparse feature fidelity (SFF) was proposed by H. Wen et.al [23] for IQA is shown in (17-19). The SFF index is computed by processing luminance correlation and feature similarity of image. To increase the accuracy and stability, two approaches, visual attention, and visual threshold were calculated to remove the perceptually insignificant information from image patches. The SFF index is computed by merging $S F F_{m}$ and $S F F_{f}$ into a quality score to remove the perceptually insignificant information from image patches. The SFF index is computed by combining $S F F_{m}$ and $S F F_{f}$ into a quality score shown in (17)

$$
S F F=\lambda \cdot S F F_{m}+(1-\lambda) \cdot S F F_{f}
$$

where $0<\lambda<1$ is a parameter for fine-tuning the relative importance of the two components.

Table 4 VSI outcomes of different algorithms

\begin{tabular}{cccccccc}
\hline BSD IMAGE & RESIHE & MMSICHE & SSR & MSRCR & MSRCP & Fuzzy \\
\hline 10081 & 0.98922 & 0.99109 & 0.94338 & 0.94471 & 0.92706 & 0.82369 & 0.99826 \\
202000 & 0.98929 & 0.96197 & 0.95525 & 0.9632 & 0.92291 & 0.7924 & 0.99793 \\
202012 & 0.99554 & 0.99149 & 0.89901 & 0.90622 & 0.89824 & 0.64404 \\
216066 & 0.97708 & 0.98043 & 0.94656 & 0.9448 & 0.92027 & 0.7766 & 0.99854 \\
226022 & 0.94991 & 0.98304 & 0.95708 & 0.9432 & 0.92566 & 0.78328 & 0.99871 \\
299086 & 0.98133 & 0.95396 & 0.9417 & 0.96906 & 0.92583 & 0.84497 \\
361010 & 0.93996 & 0.79269 & 0.93116 & 0.93116 & 0.9098 & 0.78064 & 0.99801 \\
37073 & 0.92009 & 0.94648 & 0.96863 & 0.9688 & 0.93175 & 0.82462 & 0.99789 \\
Average & $\mathbf{0 . 9 6 7 8 0 2 5}$ & $\mathbf{0 . 9 5 0 1 4 3 7 5}$ & $\mathbf{0 . 9 4 2 8 4 6 2 5}$ & $\mathbf{0 . 9 4 6 3 9 3 7 5}$ & $\mathbf{0 . 9 2 0 1 9}$ & $\mathbf{0 . 7 8 3 7 8}$ & $\mathbf{0 . 9 9 8 2 9 3 7 5}$ \\
\hline
\end{tabular}

D. Similarity Measurement

Lastly, the feature similarity component of SFF, $S F F_{f}$, is given by:

$$
\mathrm{SFF}_{\mathrm{f}}=\frac{1}{K . M} \sum_{i=1}^{K} \sum_{j=1}^{M} \frac{2 A_{i j}^{t h}+C}{\left(A_{i j}^{t h}\right)^{2}+\left(B_{i j}^{t h}\right)^{2}+C}
$$

where $K$ signifies the number of the taken feature vectors in an input image, $A_{i j}^{t h}$ and $B_{i j}^{t h}$, signifies the calculated values of the $i$-th column and $j$-th row in in $\mathrm{A}^{\text {th }}$ and $\mathrm{B}^{\text {th }}$, correspondingly. Here, a small positive constant $\mathrm{C}$ is applied to smooth out the outcome.

\section{E. Correlation Measurement}

Subsequently the selection step, the component of luminance correlation, $S F F_{m}$, that is computed on the foundation of $\mathrm{m}^{\text {ref }}$ and $\mathrm{m}^{\text {dis }}$, is defined as

$$
S_{F} F_{m}=\frac{\sum_{i=1}^{k}\left(\left(m_{i}^{r e f}-\mu\left(m^{r e f}\right)\right) \cdot\left(m_{i}^{d i s}-\mu\left(m^{d i s}\right)\right)\right)+C_{m}}{\sqrt{\sum_{i=1}^{k}\left(( m _ { i } ^ { r e f } - \mu ( m ^ { r e f } ) ) ^ { 2 } \cdot \sum _ { i = 1 } ^ { k } \left(\left(m_{i}^{d i s}-\mu\left(m^{d i s}\right)\right)^{2}+C_{m}\right.\right.}}
$$


Table 5 HaarPSI outcomes of different algorithms

\begin{tabular}{llllllll}
\hline BSD IMAGE & RESIHE & MMSIHE & SSR & MSRCR & MSRCP & FUZZY & Proposed \\
\hline 10081 & 0.9251 & 0.93162 & 0.71123 & 0.6594 & 0.44607 & 0.089533 & 0.98352 \\
202000 & 0.92375 & 0.79752 & 0.64922 & 0.65897 & 0.49724 & 0.067444 & 0.98493 \\
202012 & 0.97725 & 0.94644 & 0.55161 & 0.56962 & 0.42139 & 0.045234 & 0.99066 \\
216066 & 0.86978 & 0.89746 & 0.60996 & 0.70629 & 0.52372 & 0.07832 & 0.9914 \\
226022 & 0.68395 & 0.90921 & 0.64976 & 0.58025 & 0.46124 & 0.07426 & 0.98789 \\
299086 & 0.85738 & 0.79139 & 0.72014 & 0.7084 & 0.60544 & 0.087385 & 0.97858 \\
361010 & 0.77224 & 0.73479 & 0.65328 & 0.55152 & 0.47388 & 0.067554 & 0.98763 \\
37073 & 0.5988 & 0.67552 & 0.82737 & 0.82742 & 0.66293 & 0.15782 & 0.99377 \\
Average & $\mathbf{0 . 8 2 6 0 3 1}$ & $\mathbf{0 . 8 3 5 4 9 3 7 5}$ & $\mathbf{0 . 6 7 1 5 7 1 3}$ & $\mathbf{0 . 6 5 7 7 3 3 8}$ & $\mathbf{0 . 5 1 1 4 8 8 8}$ & $\mathbf{0 . 0 8 3 4 4 3 7 5}$ & $\mathbf{0 . 9 8 7 2 9 7 5}$ \\
\hline
\end{tabular}

where $C_{m}>0$ is a small constant to avoid dividing by zero (e. g., $\left.C_{m}=0.001\right), m_{i}^{\text {ref }}$ and $m_{i}^{\text {dis }}$ signifies the $i$-th elements of $\mathrm{m}^{\text {ref }}$ and $\mathrm{m}^{\mathrm{dis}}$, respectively.

From the study of SFF values obtained in Table 3, it is observed that the proposed method gives the highest SFF values for all images. The average SFF value shown in the bold italic face of the proposed method is 0.99779 , which demonstrates the best outcome with respect to the other algorithms. The second-best result for average SFF is given by MMSICHE algorithm which gives average SFF value as 0.96342 , is indicated by underline.

\section{F. Visual saliency-induced index (VSI)}

The visual saliency-induced index (VSI) image quality metric was proposed by Zhang [26] shown in equation 20. Here, $f_{1}$ is represented as the original image and $f_{2}$ is represented as an enhanced image. Visual saliency (VS) for $f_{1}(\mathrm{y})$ and $f_{2}(\mathrm{y})$ is signified as $\mathrm{VS}_{1}$ and $\mathrm{VS}_{2}$, correspondingly.

$S(\mathrm{y})$ shows a local similarity of $f_{1}(\mathrm{y})$ and $f_{2}(y)$ at pixel location y. $V S_{m}(y)$ is calculated as the maximum of $\operatorname{VS}_{1}(y)$ and $\mathrm{VS}_{2}(y)$. VSI metric between image $f_{1}$ and $f_{2}$ is defined as

$$
\mathrm{VSI}=\frac{\sum_{y \in \Omega} S(\mathrm{y}) \cdot \mathrm{VS}_{m}(\mathrm{y})}{\sum_{y \in \Omega} \operatorname{VS}_{m}(\mathrm{y})}
$$

Here, the spatial domain is allocated by $\Omega$. Matlab simulation results obtained from Table 4 shows the VSI results of different algorithms. It is found that the proposed method produces the highest VSI values for all images, shown in bold italic face. The average VSI value of the proposed method is 0.99829 , which is nearly equal to 1 , this is the best outcome among the exiting versions of histogram equalization methods. The second-best result for average VSI is 0.96780 given by RESIHE algorithm, which is stated by underline.

\section{G. The Haar-wavelet based perceptually similarity index}

The Haar-wavelet based perceptually similarity index (HaarPSI) [25] to color images is specified in 21 as

$$
\operatorname{HaarPSIC}_{f_{1}, f_{2}}=1_{\beta}^{-1}\left(\frac{\sum_{i} \sum_{k=1}^{3} H S_{f_{1} f_{2}}^{k}[\mathrm{i}] \cdot \mathrm{W}_{f_{1}^{\prime} f_{2}{ }^{\circ}}^{k}[\mathrm{i}]}{\sum_{i} \sum_{k=1}^{3} \cdot \mathrm{W}_{f_{1}^{\prime} f_{2}^{\circ}}^{k}[\mathrm{i}]}\right)^{2}
$$

With $H S_{f 1, f 2}^{1}$ and $H S_{f 1, f_{2}}^{2}$ expressed in (22)

Two gray level images $f_{1}, f_{2} \in \ell^{2}(\square)^{2}$, HaarPSI is computed by using the local similarity measure which is grounded on the first two phases of a two-dimensional discrete Haar wavelet transform and specified as

$$
H S_{f_{1} f_{2}}^{k}[x]=l_{\alpha}\left(\frac{1}{2} \sum_{j=1}^{2} S\left(\left|\left(\mathrm{~g}_{j}^{(k)} * f_{1}\right)[x]\right|,\left|\left(\mathrm{g}_{j}^{(k)} \times f_{2}\right)[x]\right|, \mathrm{Cf}\right)\right)
$$

where $\mathrm{Cf}>0, \mathrm{k} \in\{1,2\}$ picks either horizontal or vertical Haar wavelet filters. S denotes the similarity measure, and a two-dimensional convolution operator is denoted by $*$, more detail in [25].

HaarPSI outcomes are shown in Table 5 and it is observed that the proposed method gives the highest HaarPSI value as 0.98729 , specified in the bold italic face. The second-best result is given by MMSIHE algorithm, for which the average HaarPsi value is 0.83549 , designates through underline. It is seen that the proposed method gives the best result compared with other algorithms for every image. 
Table 6 GMSD outcomes of different algorithms

\begin{tabular}{cccccccc}
\hline BSD Image & RESIHE & MMSICHE & SSR & MSRCR & MSRCP & Fuzzy & Proposed \\
\hline 10081 & 0.32532 & 0.034332 & 0.078197 & 0.088166 & 0.25737 & 0.35637 & 0.017765 \\
202000 & 0.26554 & 0.070328 & 0.10414 & 0.10223 & 0.17339 & 0.36123 & 0.0088385 \\
202012 & 0.91487 & 0.26015 & 0.13396 & 0.13629 & 0.18873 & 0.24588 & 0.0026027 \\
216066 & 0.40872 & 0.042144 & 0.099394 & 0.088613 & 0.17659 & 0.31537 & 0.002666 \\
226022 & 0.96994 & 0.042353 & 0.090324 & 0.13175 & 0.26139 & 0.36791 & 0.0059368 \\
299086 & 0.040998 & 0.074031 & 0.032127 & 0.050478 & 0.15432 & 0.29943 & 0.0050076 \\
361010 & 0.12182 & 0.14438 & 0.10993 & 0.11537 & 0.14638 & 0.3296 & 0.0058757 \\
37073 & 0.17851 & 0.14851 & 0.061955 & 0.062016 & 0.11755 & 0.3209 & 0.0023226 \\
AVERAGE & $\mathbf{0 . 4 0 3 2 1 4 7 5}$ & $\mathbf{0 . 1 0 2 0 2 8 5}$ & $\underline{\mathbf{0 . 0 8 8 7 5 3 3 7 5}}$ & $\mathbf{0 . 0 9 6 8 6 4 1 2 5}$ & $\mathbf{0 . 1 8 4 4 6 5}$ & $\mathbf{0 . 3 2 4 5 8 6 2 5}$ & $\mathbf{0 . 0 0 6 3 7 6 8 6 3}$ \\
\hline
\end{tabular}

\section{H. Gradient magnitude similarity deviation (GMSD)}

Gradient magnitude similarity deviation (GMSD) [29] is a full-reference image quality metric. GMSD defined image quality. Let, $X$, be the original image. GMSD is computed as the standard deviation of gradient magnitude similarity (GMS) of each pixel in the image. The GMS is shown in equation 23.

$$
G M S(t)=\frac{2 M_{x}(t) \cdot \mathrm{M}_{v}(t)+\mathrm{k}}{M_{x}^{2}(t) \cdot M_{y}^{2}(t)+\mathrm{k}}
$$

Here, $\mathrm{k}$ is a positive constant. $M_{x}$ and $M_{y}$ are the magnitudes of the gradients at each pixel location $t$. GMSM calculation is shown in 24, which is utilized to compute GMSD. The total number of pixels in the image is represented by $Q$. The GMSD is defined in 25 .

$$
\begin{gathered}
\left.G M S M=\frac{1}{Q} \sum_{t=1}^{Q}(G M S)(t)\right)^{2} \\
G M S D=\sqrt{\frac{1}{Q} \sum_{t=1}^{Q} G M S(\mathrm{t})-G M S M^{2}}
\end{gathered}
$$

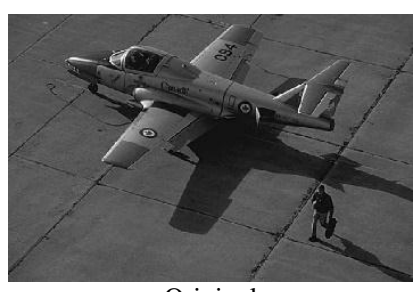

Original

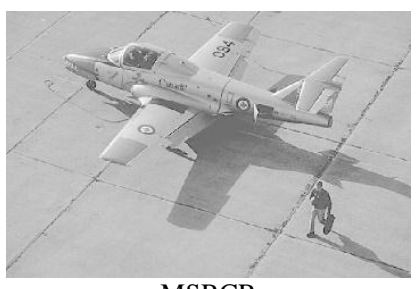

MSRCR

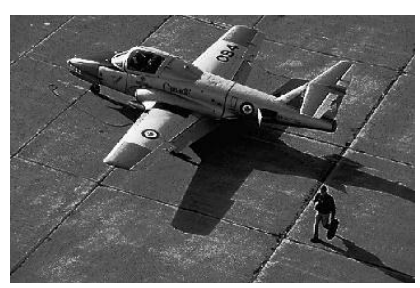

MMSICHE

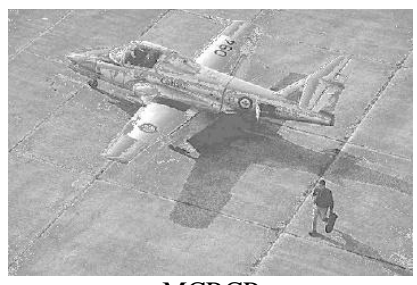

MCRCP

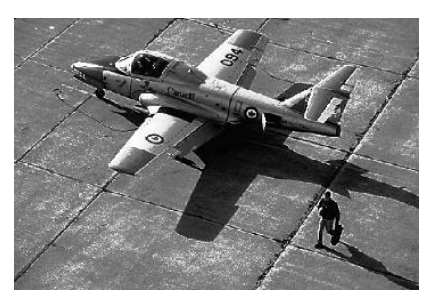

RESHIE

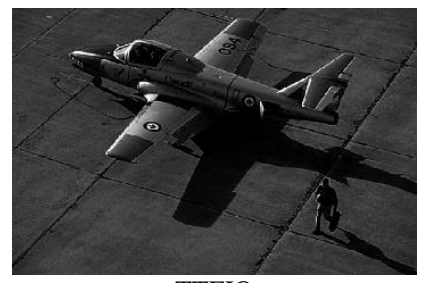

TTFIO

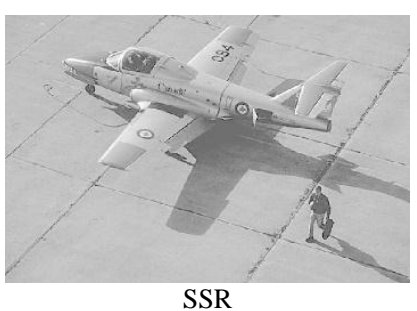

SSR

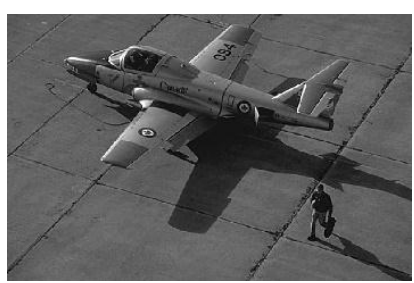

PROPOSED

Fig.4 Original BSD image '37073' of 'airplane' and output of different contrast-enhancement algorithms

GMSD can find image distortion and a lower GMSD value shows a better efficiency of visual perception. GMSD results are shown in Table 6 and it is observed that the proposed method gives the lowest GMSD value as 0.00637, demonstrated in bold italic face. The second-best result is given by SSR algorithm, for which average GMSD value is 0.08875. It is seen that the proposed method gives the best result compared with other algorithms for every image. 


\section{Visual Quality Assessment}

The Matlab simulation has been done on numerous images of the BSD image dataset [19]. Fig.4 illustrates the original image ' 37073 ' of 'airplane' and output generated from numerous contrast-enhancement algorithms. MMSICHE algorithm displays some artifacts in the ground area of the image. It is observed that R-ESIHE get over enhancement in the ground region and produces some noise in the image. SSR method gives a lighter and faded output image. MSRCR method gives almost the same result as the SSR method and produces blurry images. MSRCP method gives hazy and extra artifacts. TTFIO method gives a darker output image compared to MMSICHE and RESHIE method. The proposed method produces a smooth image with a visually better appearance as it gives improved image contrast and brightness. It is noticeable from all the enhanced images that the proposed method gives the best quality of image compared to the image produced by other methods.

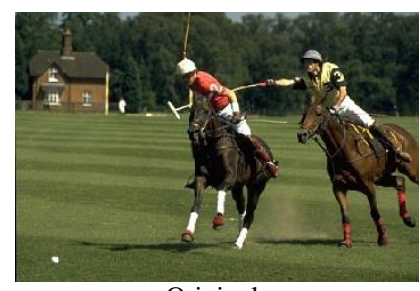

Original

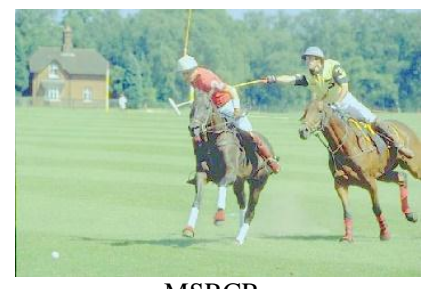

MSRCR

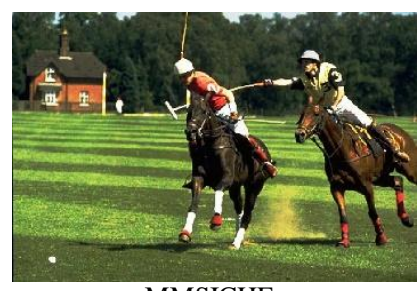

MMSICHE

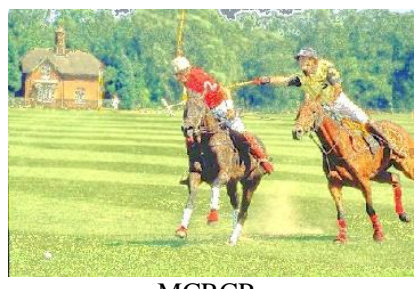

MCRCP

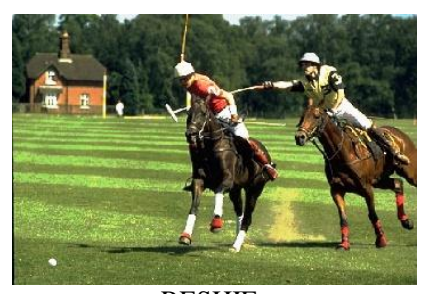

RESHIE

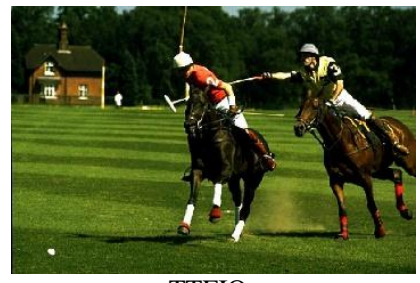

TTFIO
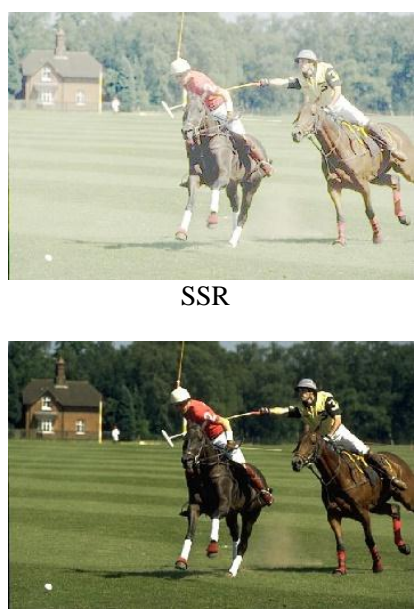

PROPOSED

Fig.5 Original BSD image '361010' of 'Polo game' and output of different contrast-enhancement algorithms

Fig. 5 displays the original image ' 361010 ' of the 'Polo game' and the results of different images obtained by different enhancement methods. Most of the grass regions get over enhancement in MMSICHE algorithm and the original color has lost at some portion in the grass. Some artifacts are shown in the background house roof. R-ESIHE get over enhancement in most of the grass regions and displays a blur effect on the dust region on the bottom side of the two horses.

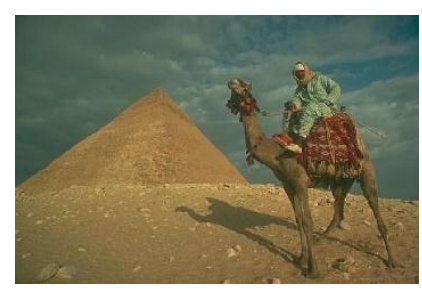

Original

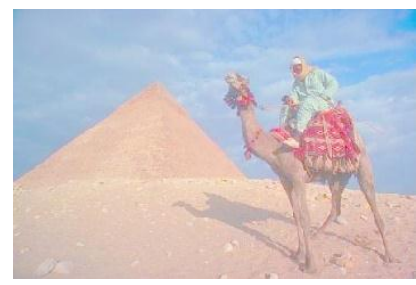

MSRCR

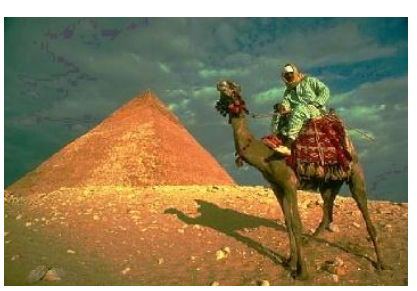

MMSICHE

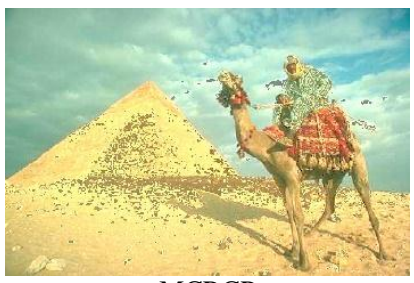

MCRCP

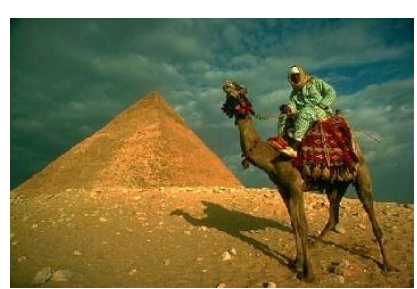

RESHIE

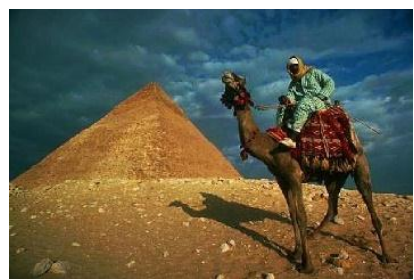

TTFIO

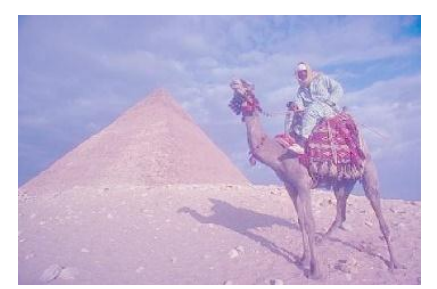

SSR

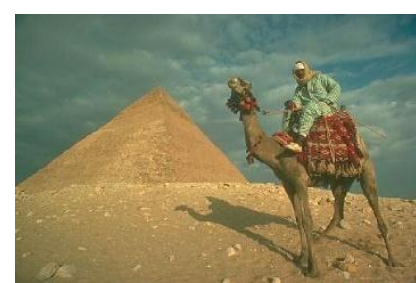

PROPOSED

Fig.6 Original BSD image '299086' of 'The Egyptian pyramids' and output of different contrast-enhancement algorithms

SSR method generates a blurry and lighter image. MSRCR produces almost the same result as SSR but shows a slightly better color. MSRCP method produces a blurry, and lighter image with extra artifacts. TTFIO method gives slightly better output with extra dark regions in the grass area. The proposed method produces an image with natural color, contrast, and brightness. 
In Fig. 6 color image '99086' of 'The Egyptian pyramids' and outcomes of images obtained by different enhancement methods. MMSICHE algorithm shows some artifacts in the sky area and the originality of color has lost out at some portion of the pyramid. R-ESIHE get over enhancement in some portion of the pyramids and shows some dark regions on the ground. SSR method produces a blurry and lighter image with changed color. MSRCR gives a blurred and lighter output. MSRCP method produces a blurred and hazy result. TTFIO

method produces some dark regions on ground and the original sky color has changed. The proposed method produces an image with better enhancement.

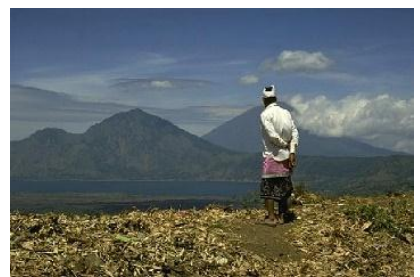

Original
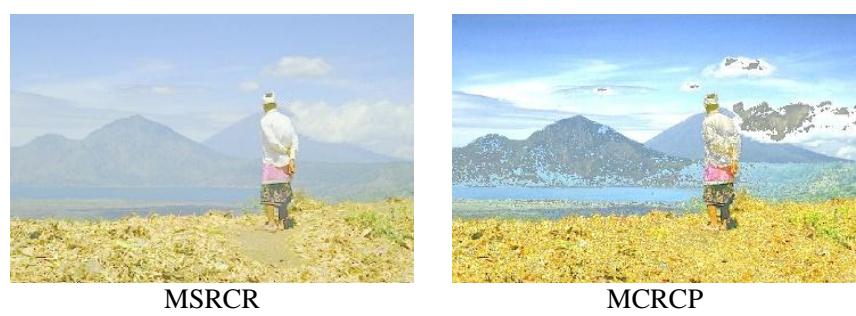

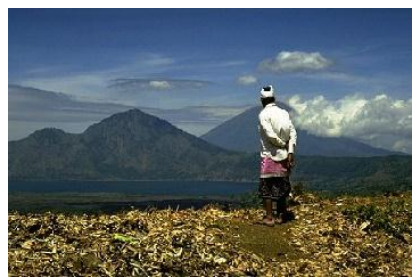

RESHIE

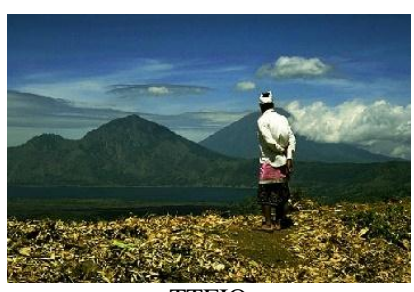

TTFIO
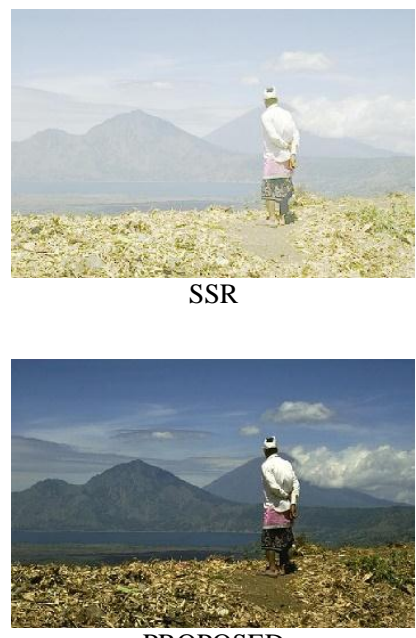

PROPOSED

Fig.7 Original BSD image '202000' of 'Mountain' and output of different contrast-enhancement algorithms

Fig. 7 shows image '202000' of 'Mountain' and output produced by different contrast-enhancement algorithms. MMSICHE algorithm shows some artifacts in the sky area. The image looks good by RESIHE method but some portion of the ground area gets effected by extra dark pixels. SSR and MSRCR return a lighter and blurry image. MCRCP gives some artifacts and noise in the sky and mountain area. The proposed method returns well-enhanced output with vibrant details.

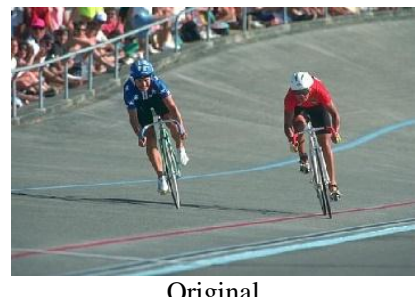

Original

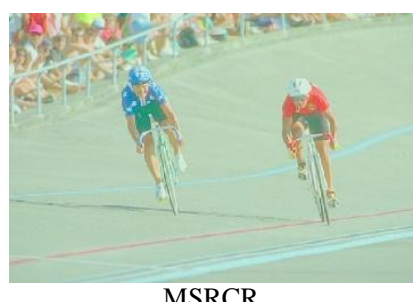

MSRCR

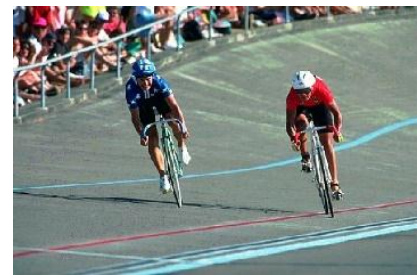

MMSICHE

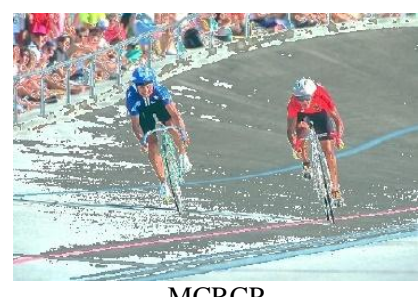

MCRCP

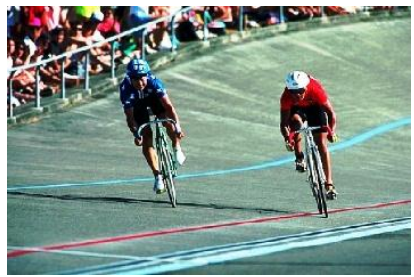

RESHIE

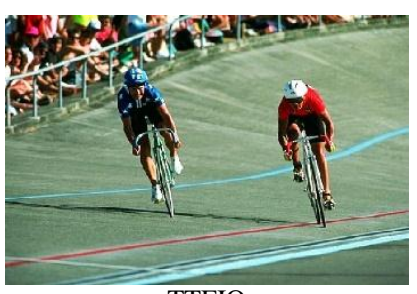

TTFIO
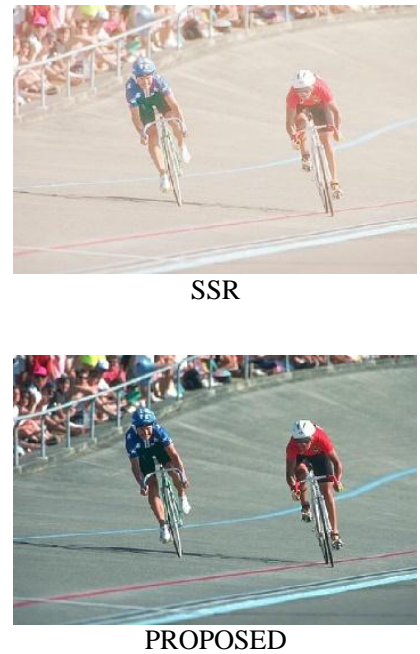

Fig.8 Original BSD image '226022' of 'Road bicycle racing' and output of different contrast-enhancement algorithms

In Fig. 8 the original color image '226022' of 'Road bicycle racing' and the processed enhanced images demonstrated by different algorithms. MMSICHE algorithm shows some artifacts in the left and bottom regions of the image.

RESIHE gives better-enhanced output than the former methods but it shows many dark regions in the road and bicycle rider portion. SSR and MSRCR methods produce a hazy, lighter and blurred image. The original color has changed in SSR methods. MSRCP method produces an image with noise, lost color, blurry with extra artifacts and hazy 
result. TTFIO method produces dark pixels in most of the image region. The proposed method shows a well visualized enhanced image over the other methods.

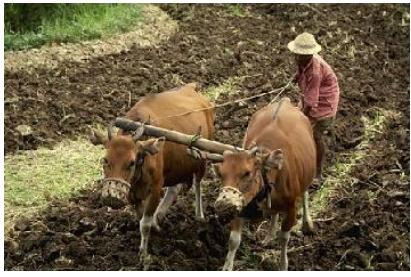

Original

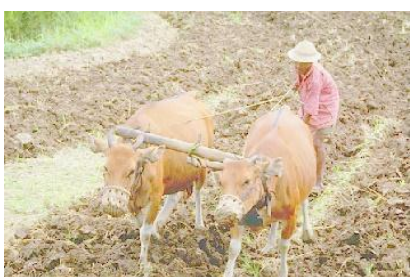

MSRCR

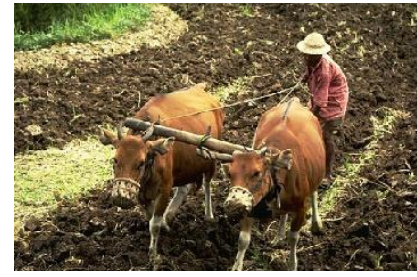

MMSICHE

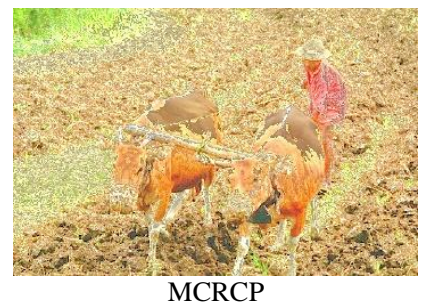

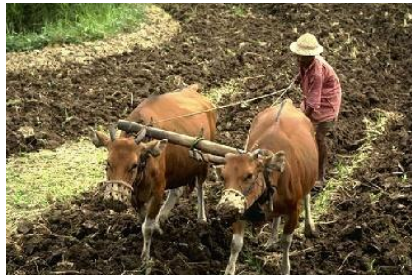

RESHIE

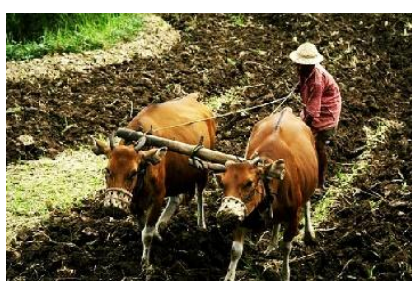

TTFIO

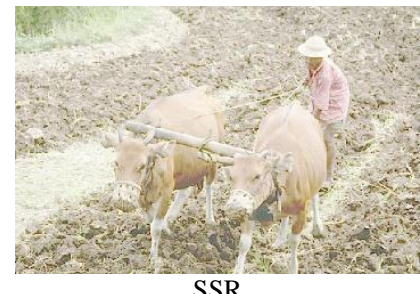

SSR

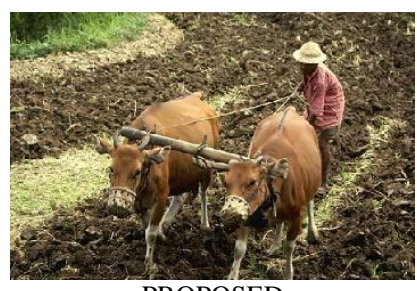

PROPOSED

Fig.9 Original BSD image '202012' of a 'Cow ploughing' and output of different contrast-enhancement algorithms

Fig. 9 exhibits the original color image '202012' of a 'Cow ploughing' and results of different images obtained by different enhancement methods. MMSICHE algorithm produces contrast enhancement but most of the grass regions get over enhancement and natural appearance of the color has been lost. R-ESIHE produces better results than MMSICHE and other existing methods but has some over enhancement in the grass area. SSR and MSRCR methods generate a blurred, hazy and lighter image and have lost the original color. MSRCP method produces an image with noise, lost color, blurry with extra artifacts and hazy result. TTFIO method produces dark pixels in most of the image region. The proposed method produces an image with natural enhancement in the context of contrast, brightness, and color. It is observed from the results that proposed method returns the best image compared to images produced by other methods.

It is observed from the results shown in Table 7, that the average computation time of the proposed algorithm is 1.66 seconds, MMSICHE runtime is 3.64 seconds and RESIHE simulation time is 4.73 seconds. It is detected from the results that our proposed skewness and mode based bi-histogram method is faster compared to mean, median and standard deviation-based methods i.e., MMSICHE and RESIHE.

Table 7 Average computation time of algorithms in seconds

\begin{tabular}{cccccccc}
\hline Algorithm & MMSICHE & RESIHE & TTFIO & SSR & MSRCR & MSRCP & PROPOSED \\
\hline $\begin{array}{l}\text { Average computation } \\
\text { time in seconds }\end{array}$ & 3.64 & 4.73 & 0.25 & 1.15 & 1.16 & 1.16 & 1.66 \\
\hline
\end{tabular}

It is also observed from the results that the average runtime of TTFIO is 0.25 seconds, SSR runtime is 1.15 seconds and MSRCR runtime is 1.16 seconds but from the results, it is observed that quantitative performance of these existing methods is poor compared to our proposed method.

Based on the simulation results, the proposed algorithm is fastest and gives the best qualitative and quantitative results over the existing state-of-art algorithms.

\section{Conclusion}

Enhancement of low contrast images are a known difficult task and such type of images are of various nature. Exploration of new easy to implement method was a scientific need and fulfillment of such requirements has been realized through execution of the proposed programmers. The claim is seen to the justified from the output results. The major use may be found out in astronomical images where having a low contrast image is a common reality. Further researches may be carried out by novel proposition of stochastic approach-based algorithms for enhancing real life images. This paper presents a new algorithm for contrast enhancement of images through skewness and mode based bihistogram equalization and subdivision of image for low contrast image enhancement. The over enhancement rate of the image is controlled by using the histogram clipping method with histogram equalization for natural enhancement. The proposed method is able to preserve the contrasts and color of the image and it improves the prominence of the lowlight effects on image regions. The present method enhances images with natural contrast and maintained image color superiority. Matlab simulation results indicate that enhanced image by proposed method carries better figure merits that 
outperform other histogram equalization-based approaches in terms of the metrics FSIM, PSIM, SFF, VSI, HaarPSI, and GMSD. These metrics values indicate better quality and robustness of the enhancement process. In the future, the proposed algorithm may be improved by processing the histogram through other statistical methods for getting enhanced image with additional features.

\section{References}

[1] Gonzalez, R.C., Woods, R.E., "Digital image processing” (Prentice-hall, NJ, USA, 2007)

[2] Chen, S. D., Ramli, A. R., "Minimum Mean Brightness Error Bi-Histogram Equalization in Contrast Enhancement", IEEE Trans. Consumer Electronics., 2003, 49, pp. 1310-1319

[3] Kim, K., Kim, S., Kim, K., "Effective image enhancement techniques for fog-affected indoor and outdoor images", IET Image Processing., 2018, 12, (4), pp. 465-471

[4] Deng, H., Sun, X., Liu, M., et al., "Image enhancement based on intuitionistic fuzzy sets theory”, IET Image Process., 2016, 10, (10), pp. 701-709

[5] Zhang, Y., Liu, H., Huang, N., et al., "Dynamical stochastic resonance for non-uniform illumination image enhancement", IET Image Proc., December 2018, 12, (12), pp. 2147 - 2152

[6] Paul, A., Bhattacharya, P., Maity, S., et al., "Plateau limit-based tri-histogram equalisation for image enhancement", IET Image Processing., 2018, 12, (9), pp. 1617-1625

[7] Al-Ameen, Zohair., "Nighttime image enhancement using a new illumination boost algorithm", IET Image Processing., 2019, 13, (8), pp. 1314-1320

[8] Tang, C., Wang, Y., Feng, H., et al., "Low-light image enhancement with strong light weakening and bright halo suppressing", IET Image Processing., 2019, 13, (3), pp. $537-542$

[9] Zarie, M., Pourmohammad, A., Hajghassem, H., "Image contrast enhancement using triple clipped dynamic histogram equalisation based on standard deviation", IET Image Processing., 2019, 13, (7), pp. 1081 - 1089

[10] Nandal, A., Bhaskar, V., Dhaka, A., "Contrast-based image enhancement algorithm using grey-scale and colour space", 2018, IET Image Processing., 12, (4), pp.514 - 521

[11] Al-Ameen, Zohair., "Visibility Enhancement for Images Captured in Dusty Weather via Tuned Tri-threshold Fuzzy Intensification Operators", International Journal of Intelligent Systems and Applications., 2016, 8, (8), pp. 10-17

[12] Matin, F., Jeong, Y., Kim, K., et al., "Color image enhancement using multi scale retinex based on particle swarm optimization method”, IOP Conf. Series: Journal of Physics: Conf. Series., 2018, 960, pp. 12026

[13] Jobson, D. J., Rahman, Z., Woodell, G. A., "A multiscale retinex for bridging the gap between color images and the human observation of scenes", IEEE Transactions on Image Processing., 1997, 6, (7), pp. 965-976

[14] Liu, Y., Yan, H., Ga, S., et al., "Criteria to evaluate the fidelity of image enhancement by MSRCR", IET Image Processing., 2018, 12, (6), pp. 880-887

[15] kansal, S., Tripathi, R.K., "Adaptive Geometric Filtering Based on Average Brightness of the Image and Discrete Cosine Transform Coefficient Adjustment for Gray and Color Image Enhancement". Arab J Sci Eng (2019)

[16] Singh, K., Kapoor, R., Sinha, S.K., "Enhancement of low exposure images via recursive histogram equalization algorithms", Optik., 2015, 126, (20), pp. 2619-2625

[17] Singh, K., Kapoor, R., "Image enhancement via median-mean based subimage-clipped histogram equalization", Optik., 2014, 125, (17), pp. 4646-4651

[18] Adelson, E.H., "Image statistics and surface perception", In Human Vision and Electronic Imaging XIII, Proceedings of the SPIE., 2008, 6806, pp. 680602-680609

[19] Sharan, L., Li, Y., Motoyoshi, I., et al., "Image statistics for surface reflectance perception”, Journal of the Optical Society of America A., 25(4), pp.846-865,2008

[20] Arbelaez, P., Maire, M., Fowlkes, C., et al., "Contour Detection and Hierarchical Image Segmentation", IEEE Transactions on Pattern Analysis and Machine Intelligence., 2011, 33, (5), pp. 898-916

[21] Zhang, L., Zhang, L., Mou, X., et al., "FSIM: a feature similarity index for image quality assessment", IEEE Trans. Image Process. 2011, 20 (8), pp. 2378-2386

[22] K. Gu, L. Li, H. Lu, X. Min and W. Lin, "A Fast Reliable Image Quality Predictor by Fusing Micro- and Macro-Structures", in IEEE Transactions on Industrial Electronics, vol. 64, no. 5, pp. 3903-3912, May 2017.

[23] H. Chang, H. Yang, Y. Gan and M. Wang, "Sparse Feature Fidelity for Perceptual Image Quality Assessment", in IEEE Transactions on Image Processing, vol. 22, no. 10, pp. 4007-4018, Oct. 2013.

[24] L. Zhang, Y. Shen, H. Li., "VSI: A Visual Saliency-Induced Index for Perceptual Image Quality Assessment", IEEE Transactions on Image Processing, 2014, 23, (10), pp. 4270-4281

[25] R. Reisenhofer, S. Bosse, G. Kutyniok and T., "Wiegand. A Haar Wavelet-Based Perceptual Similarity Index for Image Quality Assessment”. (PDF) Signal Processing: Image Communication, vol. 61, 33-43, 2018.

[26] Xue, W., Zhang, L., Mou, X., et al., "Gradient magnitude similarity deviation: a highly efficient perceptual image quality index", IEEE Trans. Image Process., 2014, 23, (2), pp. 684-695

[27] MATLAB and Statistics Toolbox Release 2018a, The MathWorks, Inc., A Natick ed., Massachusetts, United States 


\section{Authors' Profiles}

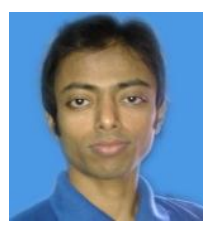

Kuldip Acharya received his M.Tech in Computer Science and Engineering from Tripura University, Tripura, India in 2012. He is doing Ph.D in Computer Science \& Engineering from National Institute of Technology Agartala, India from 2013. His research areas of interest are image processing, computer vision, and 3D Computer Animation \& design.

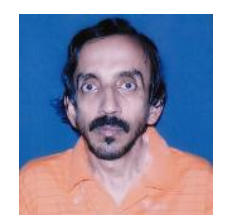

Dr. Dibyendu Ghoshal has received his B.Sc. (Honors in Physics), B.Tech \& M. Tech in Radio physics and Electronics, from Calcutta University (CU) in 1981, 1985 and 1987 respectively. He joined Indian Engineering Services (Group-A) in 1988 and served the Department of Telecommunication, GOI. He was awarded SRF in 1992 by CSIR. Subsequently, he was awarded Postdoctoral Research Associateship in 1996 and Ph.D. in Radio physics \& Electronics from CU in 1997 with a specialization in Microwave and millimeter-wave systems. His research interest includes micro \& millimeter wave, semiconductor physics \& devices, Digital Image Processing, computer vision, and computer animation.

How to cite this paper: Kuldip Acharya, Dibyendu Ghoshal, " Contrast Enhancement of Images through Skewness and Mode Based Bi-Histogram Equalization", International Journal of Image, Graphics and Signal Processing(IJIGSP), Vol.12, No.5, pp. 13-27, 2020.DOI: 10.5815/ijigsp.2020.05.02 